\title{
Pronóstico del rendimiento del IPC (Índice de Precios y Cotizaciones) mediante el uso de redes neuronales diferenciales
}

Agustín Ignacio

Cabrera Llanos

Unidad Profesional Interdisciplinaria de Biotecnología, Instituto Politécnico Nacional aicllbuda@yahoo.com

\section{Francisco}

Ortiz Arango

Escuela de Ciencias Económicas y Empresariales, Universidad Panamericana, Campus Ciudad de México fortizar@up.edu.mx

\section{Resumen}

A través de los años, el uso de las redes neuronales artificiales como herramienta de simulación, modelación y descripción de sistemas dinámicos no lineales se ha ido consolidando como una técnica eficaz y relativamente rápida gracias al gran desarrollo experimentado en los sistemas de cómputo. Esta técnica de uso común en algunas áreas de la ingeniería aplicada se empezó a utilizar en aplicaciones financieras a mediados de la década de los noventa. El presente trabajo utiliza una de las técnicas más recientes y poderosas en este ámbito: las redes neuronales diferenciales (RND), usada frecuentemente en análisis de procesos de la biotecnología. Mediante esta técnica se lleva a cabo el análisis y la estimación de la evolución del comportamiento del rendimiento del IPC de la Bolsa Mexicana de Valores (BMV) durante el periodo comprendido del 8 de noviembre de 1991 al 27 de enero de 2011. Adicionalmente, el análisis incluye un pronóstico intradía (seis valores durante una jornada de operación de la BMV) del rendimiento del IPC, el cual tiene una duración de un día después del último dato de la serie de tiempo del IPC. Cabe señalar que los resultados pronosticados mostraron una gran similitud con los datos reales.

Palabras clave: Redes neuronales artificiales, redes neuronales diferenciales, pronóstico, Bolsa Mexicana de Valores, funciones no lineales, sistemas dinámicos. 


\title{
Forecast of the IPC (Índice de Precios y Cotizaciones) return by means of differential neural networks
}

\begin{abstract}
Over the years the use of artificial neural networks as a tool for simulation, modeling and description of nonlinear dynamical systems has been consolidated as an effective and relatively fast technique thanks to the great development experienced in computer systems. This technique commonly used in some areas of Applied Engineering was first used in financial applications since the mid-nineties. This paper uses one of the most recent and powerful techniques in the field of neural networks: Differential Neural Networks Analysis (DNNA), a technique frequently used in analysis of biotechnology processes. This technique carries out the analysis and estimation of the evolution of behavior in the IPC (Stock Market Index) of the BMV (Mexican Stock Exchange) during the period from November 8, 1999 to January 27, 2011. The analysis also includes an intra-day forecast (6 values into a trading session) of the IPC return, the forecast extends during one day after the last data time series of the IPC. The predicted results showed a great similarity with actual data.
\end{abstract}

Keywords: Artificial neural networks, differential neural networks, forecast, Mexican Stock Exchange, nonlinear functions, dynamic systems.

\section{Introducción}

Desde finales de la Segunda Guerra Mundial se han venido desarrollando modelos computacionales que emulen el funcionamiento del cerebro humano (McCulloch y Pitts, 1943). Todos estos modelos tienen un elemento común: el uso de una estructura en forma de red, en la cual sus nodos (neuronas) son en realidad procesos matemáticos que a su vez incorporan los estados (resultados) de otros nodos de acuerdo con la estructura y orden en sus uniones o interacciones dentro de la red. Una clase de estos modelos son las redes neuronales artificiales (RNA), sin duda una de las más utilizadas.

El uso de las RNA como herramienta de simulación, modelación y descripción de sistemas dinámicos no lineales se ha ido consolidando como una técnica eficaz y relativamente rápida gracias a la facilidad en su uso e implementación, al gran desarrollo experimentado en los sistemas de cómputo y, lo más importante, a la capacidad que tienen para poder aproximar el comportamiento (valores) de cualquier función matemática. Las RNA poseen una gran habilidad para poder obtener 
resultados apropiados a partir de datos de entrada complejos e imprecisos y con ellos lograr identificar patrones de comportamiento, que mediante otras técnicas computacionales son muy difíciles de identificar.

Se considera que el primer modelo de una neurona artificial fue elaborado por McCulloch y Pitts (1943); en ese trabajo modelaron una estructura y funcionamiento simplificado de las neuronas del cerebro humano; el principio del diseño consistía en considerarlas como dispositivos con $n$ entradas y sólo una salida, así como dos estados posibles: activa o inactiva (McCulloch y Pitts, 1943). Debe hacerse notar que varias de las propuestas de estos autores continúan vigentes aún en nuestros días.

Una de las definiciones más aceptadas de red neuronal artificial es la siguiente:

Las redes neuronales son conjuntos de elementos de cálculo simples, generalmente adaptativos, interconectados masivamente en paralelo y con una organización jerárquica que le permite interactuar con algún sistema del mismo modo que lo hace el sistema nervioso biológico [...] (Kohonen, 1989).

\section{Figura 1}

Esquema de red neuronal estática

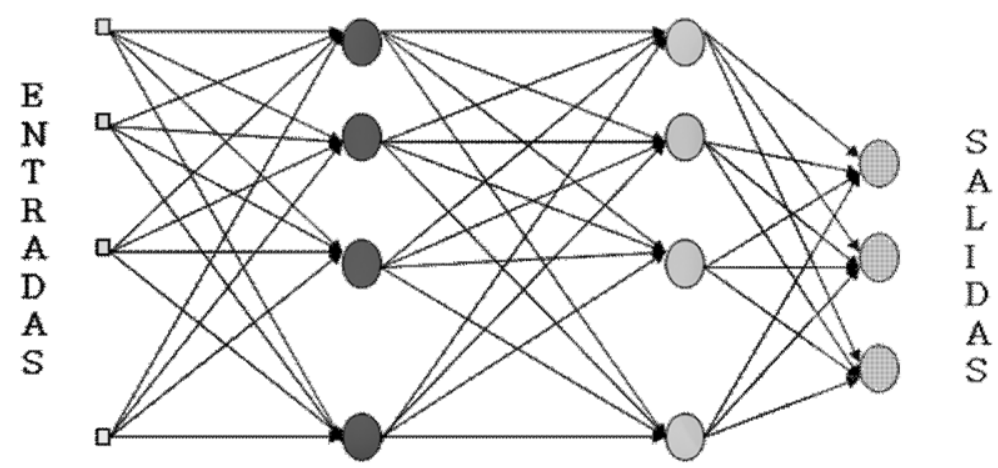

1A. CAPA OCULTA

2A. CAPA OCULTA

Los elementos fundamentales de una RNA son tres: la topología de la red, la regla de aprendizaje y el tipo de entrenamiento. 
Dadas sus características de aprendizaje adaptativo, su autoorganización y la tolerancia a fallas — que pueden operar en tiempo real y su fácil implementación en un sistema de cómputo-, han logrado que su uso se haya extendido de la ingeniería a áreas como la biológica, industrial, salud, militar y recientemente la financiera (Hilera y Martínez, 1995). Cabe destacar que esta técnica se empezó a utilizar en aplicaciones financieras a mediados de la década de los noventa.

Al tratar de analizar el comportamiento de los mercados financieros uno descubre que estos se ven afectados por factores de índole económica, política, social e incluso de naturaleza emocional; esta situación genera una interacción en extremo compleja y, por tanto, muy difícil de poder ser modelada con gran detalle $\mathrm{y}$, por consiguiente, es sumamente laborioso poder construir modelos que permitan pronosticar de manera atinada los movimientos en los precios de las acciones y las variaciones de los índices bursátiles. Los métodos que se han venido usando para hacer estos pronósticos pertenecen a una de las siguientes tres categorías: análisis fundamental, análisis técnico y el pronóstico mediante series de tiempo (Mehrara, Moeini, Ahrari y Ghafari, 2010).

El análisis fundamental (Ritchie, 1996) se basa en considerar que el precio de una acción depende de su valor intrínseco y del rendimiento anticipado sobre la inversión; para ello se deben considerar las operaciones de la empresa emisora, así como su participación en el mercado (Fama y Schwert, 1977); sin embargo, muchos autores consideran que para el caso de las especulaciones a corto y mediano plazos, no es adecuado emplear el análisis fundamental (Mehrara, Moeini, Ahrari y Ghafari, 2010).

Para el caso del análisis técnico, Murphy establece que los distintos métodos que tratan de pronosticar los movimientos de los precios futuros utilizan como referencia la información de los precios y el volumen pasados; a partir de esto, basan los métodos en la suposición de que la historia se repite una y otra vez, por lo cual el comportamiento futuro del mercado puede ser determinado analizando los datos de precios históricos (Murphy, 1999). Por otro lado, algunos autores (Coulson, 1987; Van Eyden, 1996) establecen que la mayoría de las técnicas empleadas en el análisis técnico son altamente subjetivas en su naturaleza y que carecen de validez estadística.

Asimismo, el pronóstico con series de tiempo (Box y Jenkins, 1976) se basa en técnicas estadísticas y econométricas que han sido aplicadas para predecir los mo- 
vimientos de los precios de las acciones con técnicas tales como modelos ARIMA, regresiones multivariable, o incluso utilizando relaciones de recurrencia para modelar precios históricos como funciones no lineales; posteriormente, se han usado modelos que incluyen el análisis de la volatilidad de donde proviene toda la familia de modelos ARCH, GARCH, TGARCH y otros más.

El uso de redes neuronales artificiales como una técnica de estimación del comportamiento de funciones no lineales que describen fenómenos y comportamientos financieros ha sido un gran acierto (Haykin, 1998; McNelis, 2005), también como método de estimación de precios de productos derivados (Hutchinson, 1994); otra aplicación se presentó en el cálculo de la eficiencia de pago en tarjetas de crédito (Jagielska, 1996), así como para el diseño de un portafolio de inversión (Hung, 1996). En todas estas aplicaciones, el uso de las redes neuronales ha sido de gran utilidad para llevar a cabo tanto la identificación de la serie de tiempo de los datos (Chen, 2001; Refenes, 2001), así como una técnica de estimación (Baesens, 2003; Kamruzzaman, 2003), pronóstico de precios de precios de acciones (Haykin, 1998; Mehrara, Moeini, Ahrar y Ghafari, 2010), pronóstico del índice de precios y cotizaciones de un mercado accionario (Hanias, Curtis y Thalassinos 2007).

En todos los casos señalados se puede identificar que la principal razón por la cual las redes neuronales se utilizan es el hecho de que sirven como un sistema predictor a partir de la información disponible en bases de datos tanto económicas como financieras (Kuan, 1994; Smith, 2000; Chen, 2001; Refenes, 2001). Todas las aplicaciones mencionadas tienen un punto en común: se realizaron empleando una clase de redes, las llamadas redes discretas o clásicas que utilizan preferentemente el método de propagación hacia atrás para realizar el cálculo de las ponderaciones que permiten regular su mecanismo de aprendizaje. Los resultados obtenidos con estos métodos son muy buenos y esto ha provocado que los modelos con redes neuronales emerjan como una metodología computacional que puede proporcionar nuevos elementos de análisis y descripción en aplicaciones financieras y económicas.

A partir de 1994 se han desarrollado una clase de redes neuronales artificiales basadas principalmente en el uso de las técnicas de análisis de estabilidad de acuerdo con la función de Liapunov, que se utiliza para establecer el desarrollo de las leyes de aprendizaje; estas redes son llamadas redes neuronales diferenciales $o$ dinámicas, las cuales han sido aplicadas a campos como en la biotecnología en la estimación de variables en un proceso de fermentación (Cabrera, 2002; Cabrera, 2007) utilizando esquemas de observación; a esta técnica se ha llamado sensor 
virtual (Cabrera, 2007); también en la estimación de dosis de fármacos para cáncer (Aguilar, 2006), generando una señal de control en la dosificación de tal manera que el crecimiento de células cancerosas no sea grande.

En este artículo se presentan dos resultados: un sistema o modelo de identificación y un pronóstico intradía del rendimiento del Índice de Precios y Cotizaciones (IPC) de la Bolsa Mexicana de Valores. Del sistema de identificación de la serie de tiempo de Índice de Precios y Cotizaciones (IPC) de la Bolsa Mexicana de Valores se consideró su rendimiento durante el periodo del 8 de noviembre de 1991 al 27 de enero de 2011, en el cual la red neuronal diferencial estima la evolución del rendimiento del IPC a lo largo de poco más de 19 años. Por su parte, el pronóstico intradía del IPC genera el pronóstico de seis valores del rendimiento del IPC igualmente espaciados a lo largo de un día de operación posterior al último día del que se tengan datos en la serie de tiempo del IPC. En ambos casos, tanto la descripción del rendimiento del IPC como el pronóstico de su rendimiento intradía, los resultados obtenidos son bastante alentadores debido a la precisión obtenida, lo cual induce a considerar esta técnica como una muy buena opción de análisis del movimiento de valores de índices bursátiles.

La forma en la que se organiza este trabajo es la siguiente: en la sección inicial se describe la metodología de las redes neuronales diferenciales estableciendo su descripción matemática y conceptual; después se plantea el identificador de estados, que será la base para poder construir el modelo de identificación y descripción del IPC a lo largo del periodo de tiempo establecido; más adelante se plantean los elementos empleados para poder hacer el pronóstico intradía; posteriormente, se presentan los resultados obtenidos, tanto de la identificación del comportamiento como del pronóstico intradía; finalmente, se plantean las conclusiones respecto de los resultados obtenidos.

\section{Metodología}

\section{Redes neuronales diferenciales}

La identificación de la serie de tiempo del IPC durante el periodo del 8 de noviembre de 1999 al 27 de enero de 2011 se describe en forma general como la serie de datos proporcionados por un sistema no lineal del tipo 


$$
\begin{aligned}
& \frac{d}{d t} x_{t}=\dot{\bar{x}}_{t}=f\left(x_{t}, u_{t}, t\right)+\xi_{1, t}, \text { con } x_{0} \text { conocida } \\
& \quad y_{t}=C x_{t}+\xi_{2, t} \\
& x_{t} \in \mathfrak{R}^{n}, y_{t} \in \mathfrak{R}^{p} \quad t \geq 0, u_{t} \in \mathfrak{R}^{m} \\
& C \in \mathfrak{R}^{p \times n}, \xi_{t, 1} \in \mathfrak{R}^{n \times 1} \text { y } \xi_{2, t} \in \mathfrak{R}^{p \times 1} \\
& \quad C x_{t}>\xi_{2, t}
\end{aligned}
$$

Donde $x_{t} \in \mathfrak{R}^{n}$ es el vector de estados en $t \geq 0, \quad y_{t} \in \mathfrak{R}^{p}$ es la salida correspondiente, disponible en cualquier tiempo $t$ (es una combinación lineal de los elementos de estado), $u_{t} \in \mathfrak{R}^{m}$ es la alimentación externa, $C \in \mathfrak{R}^{p \times n}$ es la matriz de transformación del estado a la salida, $\xi_{1, t}$ y $\xi_{2, t}$ son los ruidos que no pueden ser medidos (pero sí acotados) en la dinámica de los estados y la de la salida, respectivamente:

$$
\xi_{j, t} \leq \Upsilon, \Lambda_{\xi, j}>0, j=1,2
$$

que puede ser asociada con diferentes condiciones en el sistema que se analizó y los errores en la medición de la variable disponible (días de asueto distintos a sábado y domingo). Nótese que el sistema no lineal siempre puede ser descrito como:

$$
\begin{aligned}
& \dot{\bar{x}}_{t}=f_{0}\left(x_{t}, u_{t}, t \mid \Theta\right)+\widehat{f}_{t}+\xi_{1, t} \\
& \widehat{f}_{t}=f\left(x_{t}, u_{t}, t\right)-f_{0}\left(x_{t}, u_{t}, t \mid \Theta\right)
\end{aligned}
$$

Donde $f_{0}\left(x_{t}, u_{t}, t \mid \Theta\right)$ es conocida como la dinámica nominal, la cual puede ser seleccionada de acuerdo con los resultados teóricos de la red neuronal y $\hat{f}_{t}$ es el vector llamado dinámica no modelada, la cual debe ser minimizada durante el proceso de entrenamiento. Aquí el parámetro $\Theta$ es sujeto a ser ajustado para obtener la completa entonación entre la dinámica nominal y la dinámica no modelada. Por otro lado, la dinámica nominal se define como:

$$
f_{0}\left(x_{t}, u_{t}, t \mid \Theta\right)=A^{0} x+W_{1}^{0} \sigma(x)+W_{2}^{0} \phi(t) u,
$$


donde

$$
\Theta=\left[W_{1}^{0}, W_{2}^{0}\right], A^{0} \in \mathfrak{R}^{n \times n}, W_{1}^{0}, W_{2}^{0} \in \mathfrak{R}^{n \times n}, \sigma(x) \in \mathfrak{R}^{l \times 1}, \rho(t) \in \mathfrak{R}^{n \times m}
$$

Las funciones de activación $\sigma_{i}(\bullet)$ y $\phi(\bullet)$ son seleccionadas como funciones sigmoides

$$
\begin{aligned}
\sigma_{j}(t) & =a_{j}\left(1+b_{j} \exp \left(\sum_{j=1}^{n} c_{j} x_{j}\right)\right)^{-1} \\
\phi_{k l}(t) & =a_{j}\left(1+b_{k l} \exp \left(\sum_{j=1}^{n} c_{k l} x_{l}\right)\right)^{-1} \\
j & =1, n, k=1, n, l=1, m
\end{aligned}
$$

cada componente de la función de activación satisface las siguientes condiciones

$$
\sigma(x)-\sigma\left(x^{\prime}\right) \leq l_{\sigma} x-x^{\prime} ; \quad\left(\phi(x)-\phi\left(x^{\prime}\right)\right) \leq l_{\phi} x-x^{\prime}
$$

Cuya estructura es mostrada en la figura número dos.

Figura 2

\section{Esquema de red neuronal diferencial}

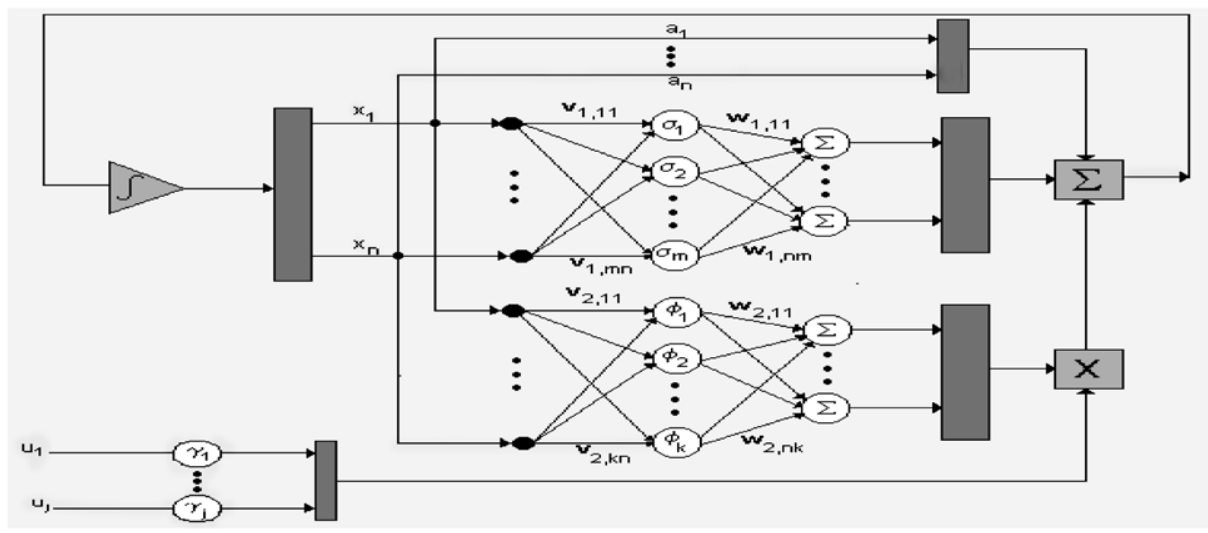




\section{Identificador de estados}

Consideremos la dinámica del lote de los rendimientos ${ }^{1}$ del IPC durante el periodo establecido anteriormente, la cual queda descrita por el sistema donde el vector de estados $\left(x_{t}\right)$ se conoce completamente (datos reales del mercado). La evolución de los estados puede ser identificada utilizando la red neuronal, buscando la mejor aproximación a este modelo no lineal usando los datos conocidos de entrada y salida. La descripción de este tipo de red neuronal es:

$$
\dot{\vec{x}}_{t}=A \hat{x}_{t}+W_{1, t} \sigma\left(\hat{x}_{t}\right)+W_{2, t} \phi\left(\hat{x}_{t}\right) u_{t}
$$

Donde $\hat{x}_{t} \in \mathfrak{R}^{n}$ es el vector de estados de la red neuronal, $u_{t} \in \mathfrak{R}^{q}$ es la acción de control, $A \in \mathfrak{R}^{n \times n}$ es una matriz Hurwitz, $W_{1, t} \in \mathfrak{R}^{n \times k}$ es la matriz de pesos para la retroalimentación de los estados no lineales, $W_{2, t} \in \mathfrak{R}^{n \times r}$ es la matriz de pesos de entrada, el vector de campo $\sigma\left(\hat{x}_{t}\right): \mathfrak{R}^{n} \rightarrow \mathfrak{R}^{k}$ es diseñado para tener elementos con una conducta de incremento monótonamente justo como función sigmoide. La función $\phi(\bullet)$ la transformación de $\mathfrak{R}^{n}$ a $\mathfrak{R}^{r \times s}$, la cual es construida por una función sigmoide de activación en cada elemento. La función de entrada $u(\bullet)$ se asume a ser acotada como $u_{t} \leq \bar{u}$. El diseño del identificador requiere de la siguiente condición: existe una matriz Hurwitz $Q$ definida positiva tal que la ecuación de Riccati tiene una solución positiva $P=P^{T}>0$ y es de la forma:

$$
A^{T} P+P A+P R P+Q=0 .
$$

\footnotetext{
${ }^{1}$ El rendimiento se define como el cociente entre la diferencia del valor actual y el valor pasado dividida entre el valor pasado: $r=\frac{P_{j}-P_{j-1}}{P_{j-1}}$.
} 
Teorema 1. Consideremos el sistema no lineal, el modelo del identificador de la red neuronal cuyos pesos son ajustados por las siguientes leyes de aprendizaje:

$$
\begin{aligned}
\frac{d}{d t} W_{1, t} & =-K_{1} P \Delta_{t} \sigma\left(\hat{x}_{t}\right)^{T} \\
\frac{d}{d t} W_{2, t} & =-K_{2} P \Delta_{t} \gamma\left(\hat{u}_{t}\right)^{T} \phi\left(\hat{x}_{t}\right)^{T} \\
\Delta_{t} & =x_{t}-\hat{x}_{t} .
\end{aligned}
$$

Siendo $W_{1,0}, W_{2,0}$ - Matrices de pesos iniciales.

Donde $K_{1}$ y $K_{2}$ son matrices definidas positivas, $P\left(P=P^{T}>0\right)$ es la solución de la ecuación de Ricatti. Se asume que la dinámica de los pesos son acotados:

$$
W_{1, t} \in L_{\infty}, W_{2, t} \in L_{\infty}
$$

y estos convergen a sus mejores valores posibles

$$
\lim _{t \rightarrow \infty} W_{1, t}=0, \quad \lim _{t \rightarrow \infty} W_{2, t}=0
$$

Además, es posible concluir que la identificación del proceso es asintóticamente consistente

$$
\lim _{t \rightarrow \infty} \Delta_{t}=0
$$

El elemento principal para construir la prueba de convergencia en este esquema es construir una función de Lyapunov para derivar las leyes de aprendizaje. Esta función de Lyapunov fue seleccionada como:

$$
\begin{aligned}
V_{t}=\Delta_{t}^{T} P \Delta_{t}+\operatorname{tr}\left[\widehat{W}_{1, t}^{T} K_{1}^{-1} \widehat{W}_{1, t}\right]+\operatorname{tr}\left[\widehat{W}_{2, t}^{T} K_{1}^{-1} \widehat{W}_{2, t}\right] \\
\widehat{W}_{j, t}=W_{j, t}-W_{j, 0}, \quad j=1, \ldots, n
\end{aligned}
$$


El procedimiento para la predicción se estableció a partir de los días previos de entrenamiento; es decir, se realizó la evolución de la red hasta cierto día especifico, el cual estaba contemplado en la base de datos; posteriormente, se realizó el cálculo de un día no establecido en los datos y que, por ende, debía calcularse su aproximación; en esta aproximación se obtuvieron seis datos entre los días de la muestra y el valor estimado. Estos datos fueron considerados como los valores intradía, que se obtuvieron de una base extra para realizar la comparación entre los datos estimados por la red neuronal; de esta comparación se obtuvieron los errores relativos y su porcentaje para comprobar la predicción hecha por la red. Estos valores son graficados de tal forma que se puede observar el error existente entre el valor real y el estimado por la red. A manera de comprobación dinámica del sistema, se grafica el índice de desempeño cuadrático para determinar la evolución de la función de error. Esto fue llevado a cabo para toda la evolución en el proceso de estimación de la red neuronal diferencial.

\section{Resultados}

La red fue construida a partir de la descripción dada por las ecuaciones (1) a (3), la programación de la misma se llevó a cabo mediante la plataforma del paquete de programación MatLab y su ToolBox Simulink, utilizando el algoritmo de Dormand Price para la solución de ecuaciones diferenciales ordinarias con paso variable. La red fue entrenada a partir de la evolución de los datos de la serie del rendimiento del IPC real (10 días de evolución de la serie), considerando los valores iniciales en las matrices de peso, las cuales fueron calculadas en un proceso de ensayo y error. A partir de este procedimiento se llegó a establecer la mejor opción para la matriz de peso inicial generando la serie de valores de error que se describen en la ecuación (3) de la ley de aprendizaje $\left(\Delta_{t}\right)$, calculados en cada iteración.

La identificación de la evolución del rendimiento del IPC y la evolución de la red se muestran en las gráficas 1,2 y 3 donde se observa que los estados se encuentran muy cercanos entre sí, esto nos está indicando que el desempeño de la red es muy bueno porque logra reproducir con gran aproximación a la serie real del rendimiento del IPC. Debemos hacer notar que la descripción de la red alcanza a estimar de manera muy aproximada incluso los cambios súbitos que experimenta el comportamiento del rendimiento real del IPC; por ejemplo, durante los días 4562 al 4565, que son mostrados en la gráfica 2, las variaciones del rendimiento diario observadas pasaron de 1 milésimo a 8 milésimos en sólo 4 días; es decir, un 
incremento del $700 \%$ en únicamente 4 días, y la red se adapta de manera inmediata al cambio en el comportamiento de la serie real.

Lo anterior, aunque no puede ser considerada una prueba de estrés, muestra el magnífico comportamiento de esta técnica ante cambios extremos. Se debe mencionar que no es el objetivo de esta metodología sujetarse a una prueba de esta índole, la cual sería propia de un análisis de valor en riesgo y no del planteamiento de este trabajo. Más adelante se comprueba con el análisis de errores mediante la integral del error cuadrático el buen desempeño de la red como método de estimación.

\section{Gráfica 1}

Valores reales y estimados por la red neuronal rendimiento del IPC

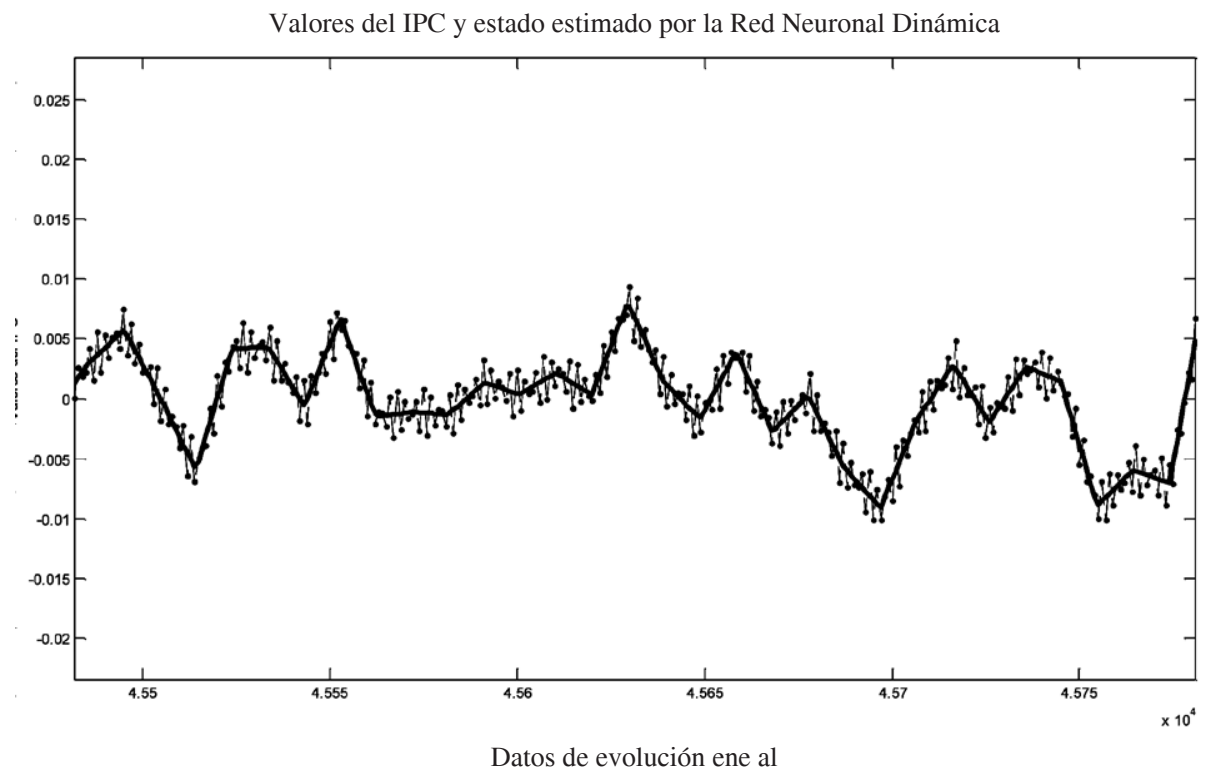




\section{Gráfica 2}

Acercamiento de los valores reales y estimados por la red neuronal rendimiento del IPC

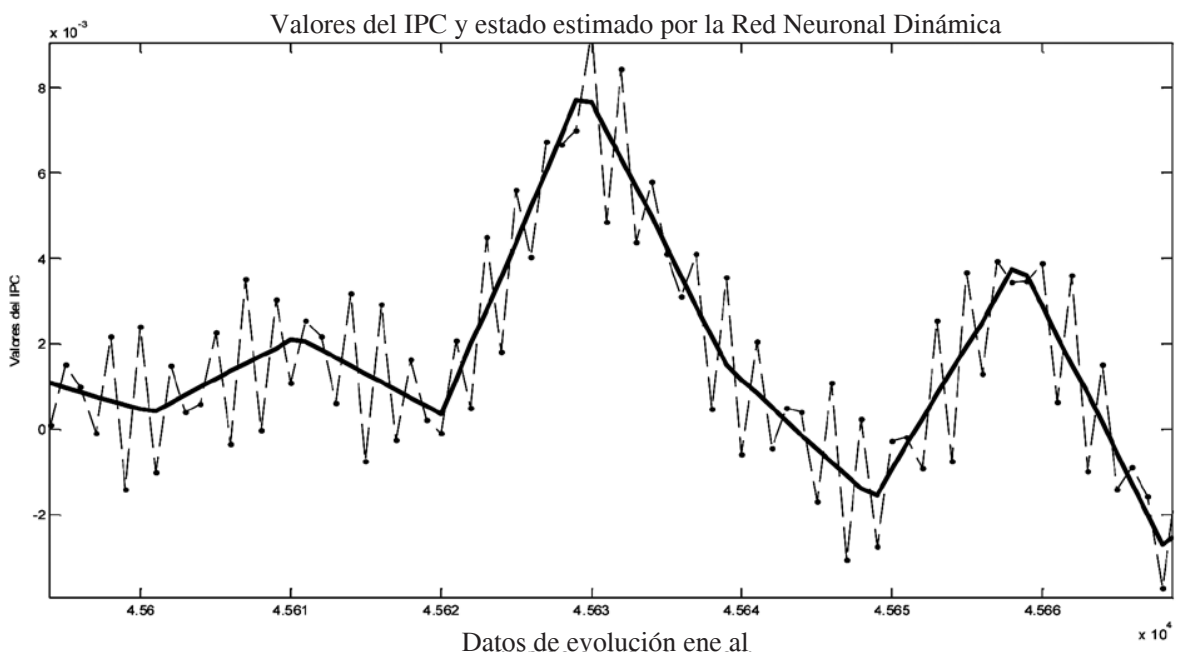

\section{Gráfica 3}

Acercamiento de los valores intradía estimados por la red neuronal del rendimiento del IPC

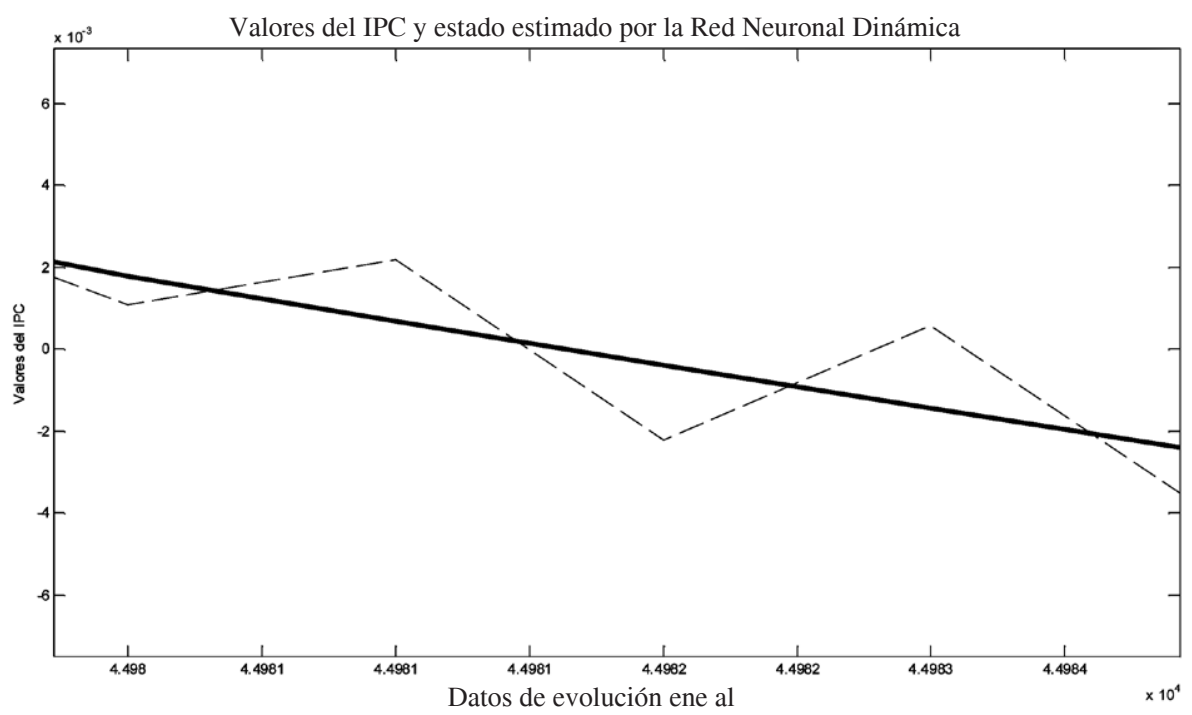


La grafica 4 muestra la evolución del índice de desempeño de la red en el cual se nota que la evolución de la integral del error cuadrático decae, indicando el alto grado de aproximación entre los estados de ambas series: la real del IPC y la generada con la red propuesta; además, se observa la variación de este valor en términos de los dígitos del eje de las ordenadas.

\section{Gráfica 4}

Evolución del Índice de Desempeño de la red

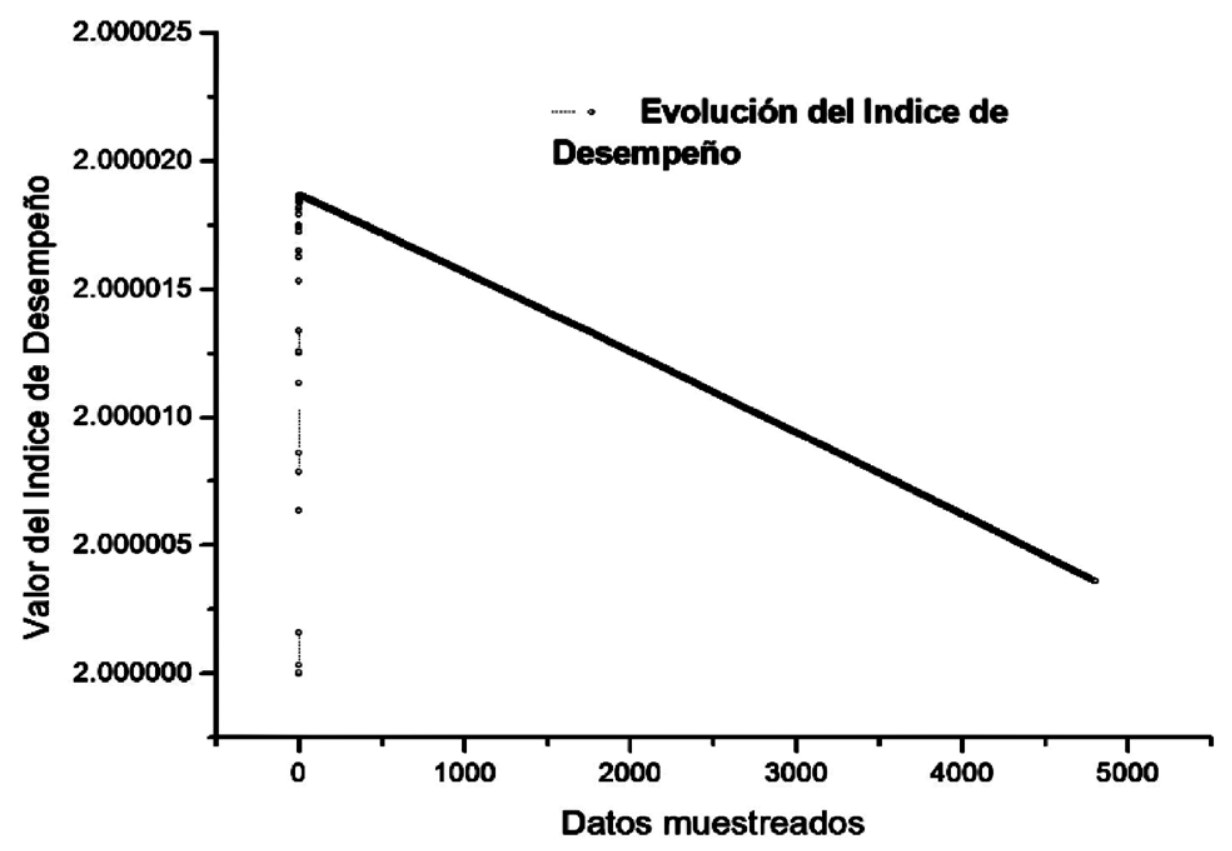

Ahora se presentan los resultados obtenidos con el pronóstico intradía del rendimiento del IPC, la fecha del pronóstico es el 28 de enero de 2011. Como puede apreciarse, las diferencias entre los valores pronosticados y los reales son mínimas, las cuales oscilan entre el $0.5 \%$ y $1.8 \%$, lo mismo ocurre con las desviaciones estándar de las diferencias entre los datos pronosticados y los reales que están comprendidas entre 0.0019 y 0.0068 . Se observa también que hay una mayor convergencia a los valores reales entre las 8:30 y las 11:06 horas de la jornada. Lo anterior se muestra en las gráficas 5a, 5b, 5c y 5d; de cuatro días de predicción. 


\section{Grafica 5}

Gráficas de los errores relativos porcentuales de las estimaciones intradía del IPC: a) 28 de enero del 2011, b) 27 de enero del 2011, c) 26 de enero del 2011 y d) 26 de enero del 2011

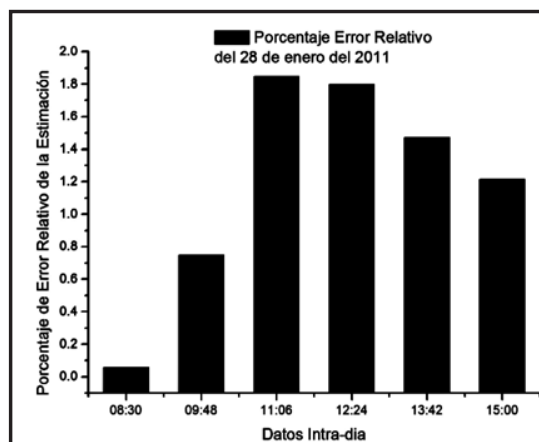

a)

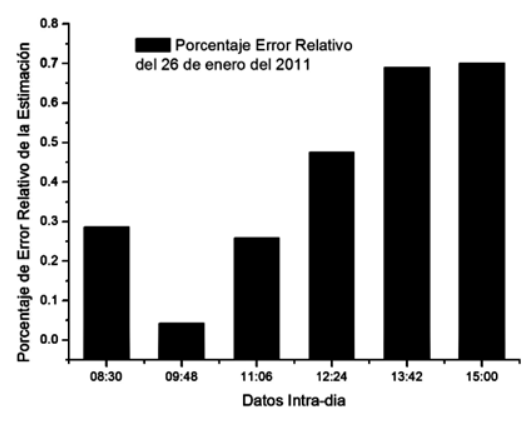

c)

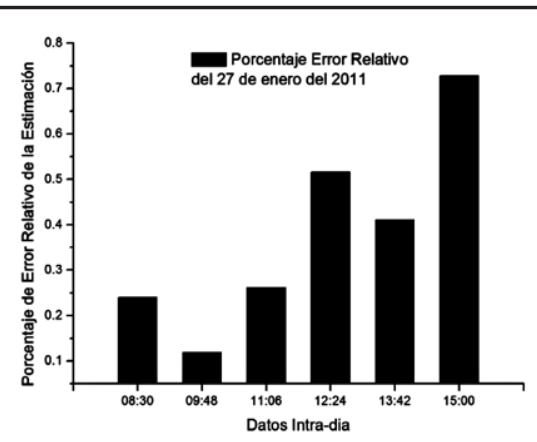

b)

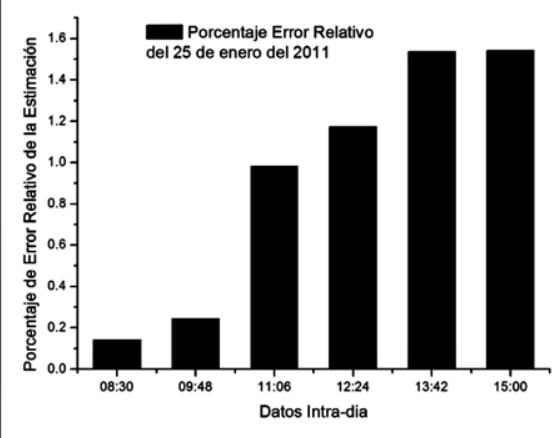

d)

\section{Cuadro 1}

Tabla de los errores relativos de la semana 1

\begin{tabular}{|c|c|c|c|c|c|c|c|}
\hline & \multicolumn{7}{|c|}{ Tabla de los errores relativos de 1 semana } \\
\hline Hora & 28 de enero & 27 de enero & 26 de enero & 25 de enero & 24 de enero & 21 de enero & 20 de enero \\
\hline $08: 30$ & 0.000563939 & 0.002399222 & 0.002857616 & 0.001425121 & 0.000216065 & 0.000321395 & 0.00188361 \\
\hline $09: 48$ & 0.007463923 & 0.001191196 & 0.000425976 & 0.0024531 & 0.003756599 & 0.000904497 & 0.007053399 \\
\hline $11: 06$ & 0.018478656 & 0.002620082 & 0.002588493 & 0.009829219 & 0.008845575 & 0.002533823 & 0.0052817 \\
\hline $12: 24$ & 0.017987027 & 0.005165133 & 0.004761703 & 0.011740006 & 0.01140566 & 0.002481187 & 0.00324997 \\
\hline $13: 42$ & 0.014723292 & 0.004110382 & 0.006902233 & 0.015359808 & 0.014251351 & 0.001349203 & 0.002451627 \\
\hline $15: 00$ & 0.012154878 & 0.00727863 & 0.007013515 & 0.015419419 & 0.016688483 & 0.002066843 & 0.003427415 \\
\hline PROM & 0.011895286 & 0.003794107 & 0.004091589 & 0.009371112 & 0.009193955 & 0.001609491 & 0.003891287 \\
\hline DESVSTD & 0.006878248 & 0.002200947 & 0.002611709 & 0.00615249 & 0.006275756 & 0.000899708 & 0.001931945 \\
\hline
\end{tabular}


Los resultados obtenidos mediante esta herramienta muestran mayor precisión que los obtenidos por Hanias, Curtis, Thalassinos (2007) para el mercado de valores de Grecia, quienes utilizaron redes neuronales artificiales con un sistema de aprendizaje backpropagation, así como por los obtenidos por Mehrara, Moeini, Ahrari y Ghafari (2010) para el mercado de valores de Irán.

\section{Conclusiones}

El sistema de identificación basado en redes neuronales diferenciales muestra un buen desempeño, lo cual permite considerar el uso de estas redes como una nueva herramienta viable, útil y robusta para la descripción precisa de variables financieras con un error de aproximación mejorado.

Por otro lado, con base en los datos de la serie de valores del IPC, se utilizó la red como un sistema de predicción por medio del cual se elaboraron estimaciones de la evolución del comportamiento de valores intradía del rendimiento del IPC de la BMV, obteniéndose valores muy cercanos a la realidad, lo que podría brindar enormes posibilidades de obtener altos beneficios en la operación diaria de compra-venta de valores bursátiles.

Se contempla como trabajo futuro el ampliar la funcionalidad de la red para convertirla en un sistema de predicción a corto plazo de 5 días y posteriormente ampliarlo hasta una predicción de 10 días.

\section{Referencias}

Cabrera-Llanos, A. I., A. P. Poznyak, T. J. S. Aranda (2002). Identification of a Fedbatch Fermentation Process: Computational and Laboratory Experiments. Bioprocess and Biosystems Engineering (24): 319-327.

T. J. S. Aranda (2007). Estimating the Treahalose Cytoplasmatic Content during a baker's yeast 10th International Symposium on Computer Applications on Biotechnology, Cancún, México, IFAC press.

M. G. R. S. (2007). Soft Sensors in biotechnology process (in spanish). Superficies y Vacío 20 (2): 1-5. 
Aguilar, N. C. e I. Chairez (2006). Neuro Tracking Control for Immunotherapy Cancer Treatment. IJCNN '06 International Joint Conference on Neural Networks. IEEE. Vancouver, BC: 5316-5323.

Baesens, B., R. Setiono, C. Mues y J. Vanthienen (2003). Using neural network rule extraction and decision tables for credit risk evaluation. Management Science (49): 312-329.

Box, G., G. Jenkins (1976). Time Series Analysis: Forecasting and Control. Holden Day.

Chen, X., J. Racine, N. R. Swanson (2001). Semiparametric ARX neural network models with an application to forecasting inflation. IEEE Transactions on neural networks (12): 674-683.

Coulson, D.R. (1987). The Intelligent Investor's Guide to Profiting from Stock Market Inefficiencies. Probus Publishing.

Fama, E., W. Schwert (1977) Asset returns and inflation . Journal of Financial Economics (5):115-146.

Hanias, M., P. Curtis y J. Thalassinos (2007). Prediction with Neural Networks: The Athens Stock Exchange Price Indicator. European Journal of Economics, Finance and Administrative Sciences, Issue 9: 21-27.

Haykin, S. (1998). Neural Networks: a Comprehensive Foundation. 2a. ed., NJ: Prentice-Hall.

Hilera, J.R. y V.J. Martínez (1995). Redes neuronales artificiales. Fundamentos, modelos y aplicaciones. Madrid: Addison-Wesley Iberoamericana.

Hung, S. Y., T. P. Liang y V. W. Liu (1996). Integrating arbitrage pricing theory and artificial neural networks to support portfolio management. Decision Support Systems (18): 301-316.

Hutchinson, J. M., W. Lo Andrew y T. Poggio (1994). A Nonparametric Approach to Pricing and Hedging Derivative Securities Via Learning Networks. Journal of Finance (49): 851-889. 
Jagielska, I. y J. Jaworski (1996). Neural network for predicting the performance of credit card accounts. Computational Economics 9 (1): 77-82.

Kohonen, T. Self-organization and associative memory. Springer Verlag, New York, 1989.

Kamruzzaman, J. y R. Sarker (2003). Forecasting of currency exchange rates using ANN: A case study. IEEE International Conference on Neural Network \& Signal Processing.

Kuan, C.-M. y H.White (1994). Artifical Neural Networks: An Econometric Perspective. Econometric Reviews (13): 1-91.

McCulloch, W.S. y W. Pitts (1943). A logical calculus of the ideas immanent in nervous activity. Bulletin of Mathematical Biology 5 (4): 115-133.

McNelis, P. D. (2005). Neural Networks in finance. San Diego: Elsevier Academic Press.

Mehrara,M., A. Moeini, M. Ahrari y A. Ghafari (2010). Using Technical Analysis with Neural Network for Forecasting Stock Price Index in Tehran Stock Exchange. Middle Eastern Finance and Economics, Issue 6: 50-61.

Murphy, J.J., 1999. Technical Analysis of the Financial Markets: a Comprehensive Guide to Trading Methods and Applications. New York Institute of Finance.

Refenes, A. P. N. y W. T. Holt (2001). Forecasting volatility with neural regression: A contribution to model adequacy. IEEE Transactions on Neural Networks 12 (4): 850-865.

Ritchie, J.C. (1996). Fundamental Analysis: a Back-to-the-Basics Investment Guide to Selecting Quality Stocks. Chicago: Irwin Professional Publishing.

Smith, K., y J. Gupta (2000). Neural networks in business: Techniques and applications for the operations researcher. Computer and Operation Research (27): 1023-1044. 
Van Eyden, R.L. (1996). The Applications of Neural Networks in the Forecasting of Share Prices. Finance and Technology Publishing. 
